\title{
Sketching expressive visualization of a natural phenomenon: Ultra-Violet individual exposure estimation
}

\author{
Laurent Moccozet $^{+}$, Alexandre Cao*, Antoine Milon ${ }^{\S}$, Pierre-Olivier Droz ${ }^{\S}$, David Vernez\% ${ }^{\%}$, \\ Jean-Luc Bulliard ${ }^{\#}$ \\ ${ }^{+}$University of Geneva (CH), *INSA Lyon (FR), ${ }^{\S}$ Institute for Work and Health (Lausanne, CH), \\ ${ }^{\%}$ French Agency for Environmental and Occupational Health Safety (Paris, FR), ${ }^{\#}$ University Institute \\ of Social and Preventive Medicine IUMSP (Lausanne, $\mathrm{CH}$ ) \\ \{Laurent.Moccozet@cui.unige.ch, Alexandre.Cao@insa-lyon.fr, Antoine.Milon@hospvd.ch, \\ Pierre-Olivier.Droz@hospvd.ch, David.Vernez@afsset.fr, Jean-Luc.Bulliard@chuv.ch\}
}

\begin{abstract}
The research presented in this paper aims at developing and validating a predictive tool of individual exposure to solar Ultra-Violet (UV). UV exposure depends on ambient irradiation level and individual factors related to activity (position to the sun, clothing, duration of exposure, and other forms of sun protection). We predict exposure levels of body parts on basis of ambient irradiation levels and information about postural activity. The prediction system uses existing techniques in the field of $3 D$ rendering to visually sketch an accurate estimation of the exposure distribution over body parts represented as a $3 D$ triangular mesh. The results are compared against individual dosimetric measurements. Our approach is based on the similarities between our assumptions about the individual UV exposure model and the rendering of $3 D$ computer generated scenes.
\end{abstract}

Keywords--- natural phenomenon, visualization, simulation, visibility, rendering, virtual human

\section{Introduction}

Excessive exposure to solar UV light is the main cause of most skin cancers in humans. Factors such as the increase of solar irradiation at ground level (due to anthropic pollution), the rise in standard of living (vacation in sunny areas), and (mostly) the development of outdoor activities have contributed to increase exposure (figure 1). Thus, unsurprisingly, incidence of skin cancers has increased over the last decades more than that of any other cancer.

This research aims at developing and validating a predictive tool of individual exposure to solar UV. Exposure levels of body parts are predicted on basis of ambient irradiation levels and information about postural activity. The prediction system uses existing techniques in the field of 3D rendering to visually sketch a rough but accurate estimation of the exposure distribution over body parts and is compared against individual dosimetric measurements. Our approach is based on the similarity of the UV exposure context with the rendering of $3 \mathrm{D}$ computer generated scenes.

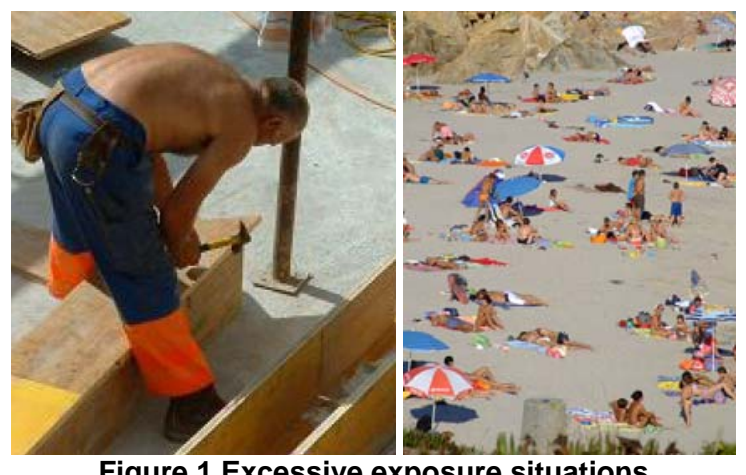

Figure 1 Excessive exposure situations

The use of numeric methods adapted from 3D rendering should facilitate assessment of individual exposure and allows predictive scenarios (either prospective or retrospective). In the long run, a better quantification of exposures for specific occupational and leisure activities should assist in better targeting preventive actions.

As raised in [30] , "applications of human modeling and simulation include many fields and industries. I name a few for your reference: medical, sports, manufacturing, military, forensic simulation, design for disability, comfort, biomechanics, exercise science, ergonomics, safety design, pain and injury, and many more. These topics range from the realistic modeling of humans-their looks, texture, and skin deformation-to the more advanced topics of behavior, motion prediction, and cognitive aspects." Human modeling and simulation is now a mature field [15] [22] [23] and apart from the film industry, it has been successfully applied in a wide range of areas: training [25] , ergonomics [21] [24] [27] , medicine [26], surgery [28] and many more [30] . Surprisingly, although a particular interest has been dedicated to the human body exposure to UV [33] [34] , very limited efforts have been brought to the application of human modeling and 3D Computer Graphics for estimating and visualizing exposure to irradiation in 
general [29] and to UV irradiation in particular [12] Precisely simulating the UV irradiation phenomenon is unrealistic. However an accurate compromise can be achieved to sketch the phenomenon by using real data measurements and combining them with simple numerical simulation techniques such as the one applied for rendering in 3D Computer Graphics.

In the next section, we will define the scientific context related to the individual exposure to UV irradiation. We will then depict our model for simulating UV exposure based on ambient irradiation measurements data in section 3.1, which will bring us to the description of the implementation of our prototype simulator in section 3.2. We will finally conclude our study by discussing our experiments and results in section 4.

\section{Scientific context}

Skin cancer is becoming a critical growing public health problem. As it can be simply reduced by prevention, more communication and information data about individual UV exposure is required in order to adapt the protection and the behavior with respect to sun exposure.

UV exposure depends on ambient irradiation level and individual factors related to activity (position to the sun, clothing, duration of exposure, and other forms of sun protection) [11]. The risk of skin cancer varies according to the skin type and the exposure of anatomical parts of the body. The neck, shoulders and head are usually the most irradiated parts.

We briefly review the effects of UV radiations, the current status of ambient radiations measurements and of exposure assessment.

\subsection{Effects of UV radiations}

Switzerland is among the European countries the most affected by this public health problem. Only $10 \%$ of all skin cancers are melanoma, the most lethal type. Most cutaneous cancers are basal and squamous cell carcinomas, for which prognosis is better. In Switzerland, more than 10'000 non-melanoma skin cancers are diagnosed every year according to estimates based on regional cancer registries.

Although less frequent (only $10 \%$ of skin cancers), melanoma is a more severe disease. Because of this, and because it affects a relatively young population (half of melanoma patients are diagnosed before the age of 60), melanoma contributes significantly to lifespan reduction. The incidence of melanoma has doubled every 10 to 15 years in white skin populations during the last 40 years [1] . In Europe, about 26'100 new cases of melanoma in men and 33'300 in women are registered each year, and about 8'300 men and 7'600 women die from this disease. Melanoma is the 8th commonest cancer for women and the $17^{\text {th }}$ most frequent cancer for men [2] . One in five melanoma patients die from it, while early diagnosis and treatment could save almost all cases [3] [4] .

\subsection{Prevention and public health}

At a population level, primary prevention activities sensitize the public to the deleterious effects of an excessive sun exposure, while secondary prevention aims at early detection and surveillance of high-risk individuals.

General irradiation indicators such as the UV Index have recently been added to the prevention tools. UV index has been developed and broadened as a didactic risk indicator to raise public awareness toward solar irradiation exposure. Until now, its impact on sun behavioral changes has however been limited [5] [6] . The important lag time between sun exposure and its observable cutaneous damage, along with the positive social perception of tanning, render compliance with the most sensible sun protective measures sometimes difficult [7] . More appropriate indicators have to be proposed in order to improve prevention. It must be pointed out that individual dosimetry has rarely been used as an evaluation tool in prevention.

As shown in an Australian study, informing people about the effects of an overexposure to the sun while taking into account the role of their activity in the daily UV dose seems to be an efficient sensitization strategy. A 33\% reduction in sun exposure was measured, mostly because of behavioral changes during the most exposed activities (using protective clothing, seeking shaded areas) when people are directly informed about UV measurements and exposure [8] .

\subsection{Assessment of ambient irradiation}

The monitoring of both the increase in skin cancer occurrence and the ozone layer depletion have increased the need of ambient irradiation data. Consequently, in situ measurements have been enhanced and generalized, and efforts have been undertaken to develop predictive irradiation models based on routine meteorological measurements. Currently, ground radiation measurement stations exist in most European countries, either using broadband detectors or spectro-radiometers.

In the context of individual exposure, 3 kinds of irradiation data are of particular interest: direct irradiation, diffuse air irradiation and irradiation reflection due to the surrounding environment (albedo). Human irradiation is usually expressed in MED (Minimal Erythemal Dose) units, which takes into account the UV action spectra (frequency weighting). MED measurement does not require a spectral analysis and may be obtained from broadband detectors having the adequate spectral response. Diffuse and reflected irradiation may also be obtained from broadband detectors equipped with a shading cache or facing ground, respectively.

\subsection{Assessing exposure}

Ambient irradiation gives little insight on the effective radiation experienced by exposed individuals. 
Depending on the time and duration of exposure, and the body position to the sun, the human body may receive 24 to $61 \%$ of the total ambient irradiation [9] . Various dosimetric techniques have been developed to assess individual exposures (figure 2), such as photo-electrical captors or photo-sensible chemicals/biologicals (e.g. polysulfone badges). However, dosimetric measurements remain tedious, as many dosimeters (about 10-20) are needed for each individual to assess exposure of various body locations. Moreover, exposure assessed by dosimetric measurements tends to be situation-specific and prone to epidemiological biases, so that their generalization remains difficult. Results obtained for a given activity can rarely be extrapolated to assess the exposure of the same activity in other ambient irradiation conditions.

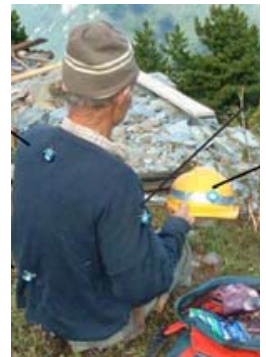

Figure 2 Individual dosimetry setup

\section{Individual UV exposure simulation}

In this study, we are applying the sketching principle to the simulation and the visualization of a natural phenomenon. Similarly to complex object shapes many natural phenomena are highly complex to simulate. However, in many situations and contexts, a precise simulation is not required. Our global approach for visualizing the natural phenomena of human body exposure to Ultra-Violet (UV) can be compared to the concept of sketching: a sketch is usually a hand-drawn figure that approximates a specific shape. Although the sketch figure is not as precise as the shape itself, it provides enough visual clues and details and depicts the shape in a way that makes it "meaningful". By meaningful, we mean that anyone is able to identify the shape by looking at the sketch and that it conveys the ideas and intentions that the author wants to transmit. The basic objective of a sketch figure is not to reproduce exactly the shape of a given object but to communicate ideas about the object. The focus of sketching is communication rather than completeness. It does not mean that the sketch is not accurate, but that the accuracy is adapted to the intention to communicate. In Computer Graphics, non-photorealistic rendering (NPR) techniques have emerged in order to introduce expressive rendering such as sketch style [10]. One application consists in producing 3-D images with enhanced visual comprehensibility. Our objective is to appropriately communicate about body exposure in order to improve prevention. The communication objectives are for example to show how UV are distributed over bodies in order to help people how to dress accurately for outdoors activities or to help clothes makers to design accurate outdoors clothes.

\subsection{Exposure model}

The model previously developed in [11] uses UV irradiation datasets measured with a radiometer. The values of irradiation measured for a specific orientation (angle with the horizontal) are then applied on each body surface with the same orientation. That means, for a given geographical position, the solar irradiation has to be measured specifically for this purpose and for the duration of the excepted exposure.

In our case, we use general continuous datasets of ambient irradiation measurements on a horizontal surface modified as a homogeneous sphere of irradiation. And then, we expose the $3 \mathrm{D}$ mannequin to this sphere of irradiation for the wished duration.

Compare to [11] it means that we are able to use UV irradiation measurements performed by meteorological stations. Therefore, it is not necessary to be on the geographical location of irradiation and it is also possible to perform a retrospective simulation of the exposure as far as the UV measurements exist.

The three components of the solar irradiation (direct, diffuse and albedo) are implemented in an existing program of human body modeling. Daily irradiation cycles were collected at the Baseline Surface Radiation Network (BSRN, SolarLight 501A UV broadband radiometers) station of MeteoSwiss at Payerne1 (located at $491 \mathrm{~m}$ above sea level, figure 3).

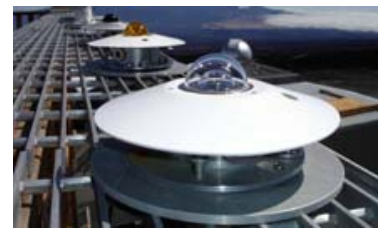

Figure 3 Radiometer for measuring ambient UV irradiation

From the daily cycles data, the solar irradiation has been modeled to incorporate the human model in the field. The diffuse $(\mathrm{D}(\mathrm{t})$ in figure 4$)$ and albedo (reflection through ground, $\mathrm{R}(\mathrm{t})$ in figure 4) components are considered as hemi-spherical homogeneous sources with the intensities varying in function of time whereas the direct component ( $\mathrm{I}(\mathrm{t})$ in figure 4) is described as a parallel source of irradiation varying in intensity with time and in direction with the position of the sun.

The human 3D model is used to represent the exposed human. The body surface is subdivided into triangles whose size depends on the whished resolution. Each triangle receives a certain amount of energy accordingly to the lightning from the solar irradiation. In function of the shadowing due to elements of the body,

\footnotetext{
${ }^{1}$ Payerne facility is part of the Baseline Surface Radiation Network (BSRN) of the WMO World Climate Research Programme.
} 
the posture and the orientation to sun, the perceived irradiation from a single triangle is expressed as a combination of exposure from the three components of the solar irradiation. The exposure of the body (expressed in $\mathrm{J}^{-\mathrm{m}^{-2}}$ ) can be visualized as the sum of the three components or as the contribution of each type of irradiation separately.

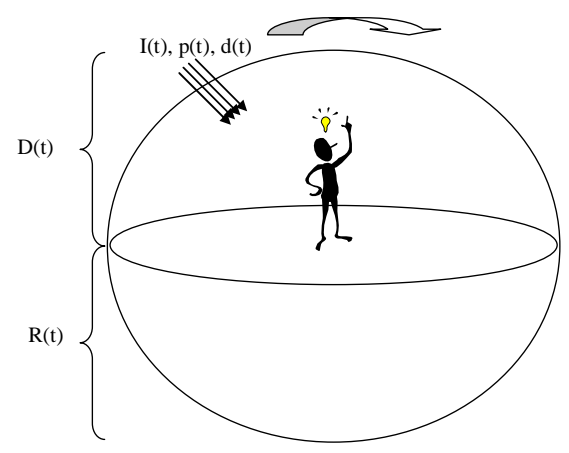

Figure 4 Solar irradiation model

\subsection{Implementation}

We have developed a program prototype implementing our hypothesis regarding the UV exposure model. The objective is to assess if the exposure model is viable for a fast and accurate approximation of UV exposure of a human body in any posture. For this purpose we use a traditional human modeling and animation approach (figure 5) based on articulated skeleton and 3D surface skinning [15] available in [20] .

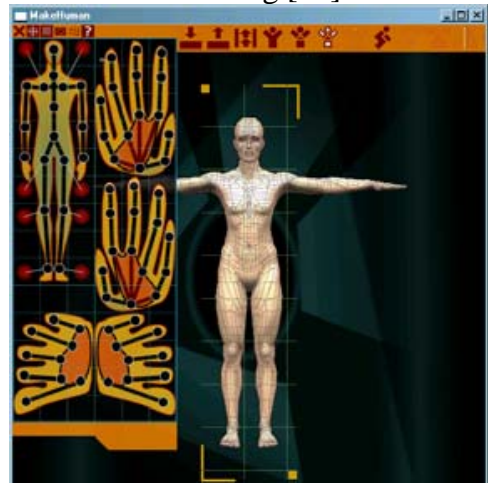

Figure 5 Human modeling and animation system

The simulation system is taking as inputs:

- the ambient UV irradiation measurements performed by meteorological stations that provide the global, diffuse, reflected and direct UV (in Watt/m2) at a step of 1 minute and the location of the sun (with the zenith and azimuth). We consider that the values are constant during the time step.

- the virtual mannequin represented as a 3D surface of connected triangles. It reproduces the shape of the real mannequin (figure 6.a) in the different postures (figures 6).

The result is the virtual mannequin with color values assigned to each vertex of the 3D mesh surface (figure 8 $\& 9)$. The color value represents the global exposure of the corresponding skin location. Intermediate values of exposure over triangles can be estimated by simply interpolating color values of the triangle vertices.

As exposed in figure 4, we compute separately the 3 exposure components (diffuse / D(t); reflected / $\mathrm{R}(\mathrm{t})$ and direct / I(t)). The main issue for the computation is related to visibility: in order to estimate how much a given vertex of the $3 \mathrm{D}$ mesh receives, we need to estimate the ratio visibility with respect to the exposure source. Visibility is a critical issue in 3D Computer Graphics [13] [14] [31] [32] that arise at many stages of the rendering pipeline such as lighting and shadow computation. We adapt state of the art visibility computation and optimization techniques in the objectspace to suit our particular context:

1. Diffuse/reflected components: according to our assumption, these components are homogeneously spread over a half-sphere. Our basic approach consists in discretizing the half-sphere into regular subsurfaces. Each sub-surface is assigned with a portion of the global component and is represented by its barycenter. For each vertex of the $3 \mathrm{D}$ mesh, we estimate how many sub-surfaces are visible from the vertex. It allows estimating the ratio of exposure that the vertex is receiving. By calculating the visibility of each vertex from the half-sphere we construct a visibility map. This map needs to be constructed only once for the whole exposure session (only the exposure value is changing over time). Visibility computations are particularly expensive and heavy in computation time and resources as it requires a lot of intersection estimations. In order to optimize the global estimation, we first optimize the amount of vertices in the virtual mannequin and we use a hierarchical subdivision space approach (we divide the box that contains the mannequin into sub-boxes; these sub-boxes are first check for intersection prior to the triangles of the $3 \mathrm{D}$ mesh) in order to reduce the number of useless intersection tests.

2. Direct component: the direct component is considered as a directional light source. For each time step, we estimate which are the vertices of the $3 \mathrm{D}$ mesh that are visible from that light source. Visible vertices receive the direct intensity.

The prototype application is developed in $\mathrm{C}++$ and based on available open source libraries and software packages: vcg [18], meshlab [17] and makehuman [16] .

1) vcg: A portable $\mathrm{C}++$ templated library for manipulation, processing of triangle and tetrahedral meshes. It includes a lot of algorithm for mesh processing task, like mesh cleaning smoothing and denoising.

2) meshlab: MeshLab is a portable, and extendible system for the processing and editing of unstructured huge $3 \mathrm{D}$ triangular meshes arising in $3 \mathrm{D}$ scanning. It provides a set of tools for editing, cleaning, healing, inspecting, and rendering.

3) makehuman: Makehuman provides a specialized application for parametrical modeling of threedimensional humanoid characters. 
Makehuman is being used to produce the virtual human mannequin in the different postures and Meshlab to post-process the 3D scan of the mannequin and the virtual human mannequin from makehuman, particularly to fill holes, smooth and reduce the geometric data. VCG is being used to implement the UV exposure estimation.

\section{Experiment and results}

A forensic mannequin (figure 6.a) has been exposed to the sunlight at the MeteoSwiss station at Payerne in different postures with individual dosimeters located at significant locations of the body shape. The resulting measurements are only used for comparison with the estimated results from the virtual mannequin. The forensic mannequin has been scanned (figure 6.b) with a full body 3D scanner in the standing posture.

The makehuman framework is being used to produce a rough $3 \mathrm{D}$ replica of the mannequin in the different postures (Figure 6.c and 7). The two 3D data sets in figures 6.b and 6.c are used to estimate the accuracy of the virtual mannequin compared to the real shape of the mannequin used for irradiation measurements. We use the ambient UV irradiation measurements performed at the meteorological station at Payern for the simulation in order to render the individual UV exposure onto the 3D mannequins.

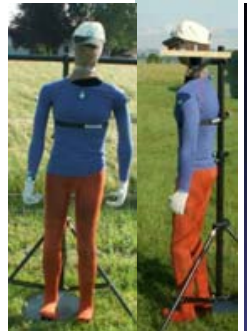

(a)

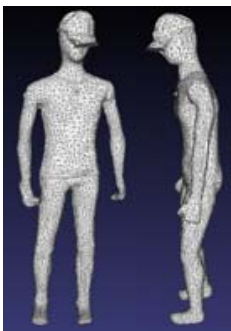

(b)

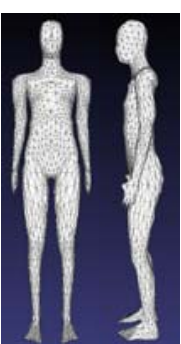

(c)
Figure 6 (a) Real mannequin (b) 3D scan of the mannequin (c) Virtual human mannequin
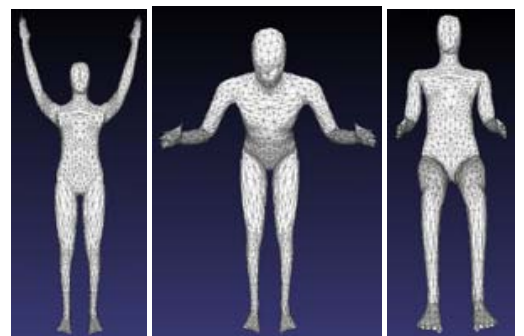

Figure 7 The virtual mannequin in different postures

In order to communicate the main information, we use simple visualization techniques based on false colors. In figure $8 \& 9$, for simplicity, the exposure value is scaled to a range of 0 to 255; values between 0 and 100 are assigned to the blue component (red and green components are set to 0 ), values between 100 and 200 to the green component (red and blue components are set to 0 ) and values above 200 to the red component (green and blue components are set to 0 ). We can check that we get consistent results on the top of the head and shoulders with the scanned mannequin and the virtual one, whereas results on the chest are different because of the cap worn by the mannequin, which is consistent.

From the validation, we can establish that the model provides satisfactory qualitative results (figure 9). The values obtained from the dosimeters and the ones computed from the model are globally consistent. They do not show any systematic deviation. However the deviation is growing for weak irradiation exposures. Moreover, the model is under estimating the irradiation exposure at the wrist. The deviations are probably linked to the difference of orientation and configuration between the dosimeter and the virtual skin shape and also to the different shapes and articulated structures of the forensic and virtual mannequins. This is particularly true when matching the different non-standing postures.
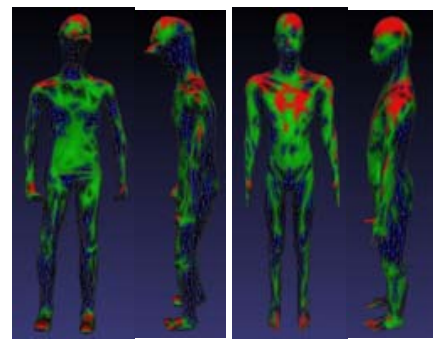

Figure 8 Visualization of global UV exposure

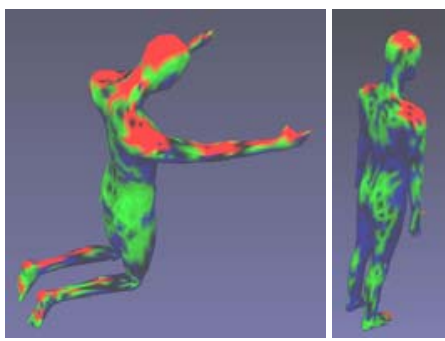

Figure 9 Variations of individual UV exposure according to postures

\section{Conclusion}

Future research and developments will aim at developing tools used in assessing individual exposure to solar UV. The proposed tool should enable to link, for any given ambient irradiation situation, factors of individual exposure to the effective dose received. The individual factors to be considered are those specific to an activity and susceptible to modify the exposure levels or exposure dose, namely: exposed skin surfaces, skin orientation to the sun, exposure duration. By linking individual factors and exposure, effective exposure (UV dose received) could be predicted for a given activity (work or leisure) in various ambient irradiation conditions. Several advantages of such a generic approach can be pointed out: as dosimetry is not systematically required any more to assess individual exposure, more assessments can be made at lower costs and prospective or retrospective assessments are also possible. Finally, objective quantification of the potential UV exposure (and risk) associated with various activities is possible; comparison of exposure between activities 
should enable to better rank at-risk activities and target sensitization messages and preventive measures.

\section{Acknowledgements}

The authors would like to thank Laurent Vuilleumier from Swiss Meteo for his advices and for the ambient irradiation data, MIRALab (www.miralab.unige.ch) at the University of Geneva for providing access to the whole body 3D scanner and to the AIM@SHAPE (www.aimatshape.net) network of Excellence for providing access to their $3 \mathrm{D}$ shape processing tools library.

\section{References}

[1] Boyle P, Maisonneuve P and Doré J-F, Epidemiology of malignant melanoma, Br Med Bull 1995, 51: 523-54.

[2] Ferlay J, Bray F and Pisani P GLOBOCAN 2000 : Cancer incidence, mortality and prevalence worldwide, IARC CancerBase no 5, Lyon, IARCPress 2001.

[3] International commission on Non-ionizing radiation protection (ICNIRP), Guidelines on limits of exposure to Ultraviolet radiation of wavelengths between $180 \mathrm{~nm}$ and 400 nm (Incoherent Optical Radiation), Health Physics Vol. 87, No 2, pp 171-186, 2004.

[4] Thompson JF, Scolyer RA, Kefford RF, Cutaneous melanoma, Lancet 2005, 365, 687-'701.

[5] Geller AC, Hufford D, Miller DR, Sun T, Wyatt SW and Reilley B, Evaluation of the Ultraviolet Index: media reactions and public response, J Am Acad Dermatol 1997, 37: 935-941.

[6] Bulliard J-L and Reeder A, Getting the message across: sun protection information in media weather reports in New Zealand, N Z Med J 2001, 114: 67-70.

[7] Autier P, Doré J-F, Reis AC, Grivegnée A, Ollivaud L and Truchetet F, Sunscreen use and intentional exposure to ultraviolet $\mathrm{A}$ and $\mathrm{B}$ radiation: a double blind randomized trial using personal dosimeters, $\mathrm{Br} \mathrm{J}$ Cancer 2000, 83: 1243-1248.

[8] Kimlin MG and Parisi AV, Usage of real-time ultraviolet radiation data to modify the daily erythemal exposure of primary schoolchildren, Photodermatol Photoimmunol Photomed, 17: 130-5, 2001.

[9] Parisi AV, Kimlin MG, Wong JC and Fleming RA, The effects of body size and orientation on ultraviolet radiation exposure, Photodermatol Photoimmunol Photomed 1996, 12: 66-72.

[10] Sayeed R and Howard T, State-of-the-art nonphotorealistic rendering (NPR) techniques, Theory and Practice of Computer Graphics 2006.

[11] Milon A, Sottas P-E, Bulliard J-L and Vernez D, Effective exposure to solar UV in building workers: influence of local and individual factors, J Expo Sci Environ Epidemiol, 17: 58-68, 2006

[12] Streicher JJ, Culverhouse Jr. WC, Dulberg MS and Fornaro RJ, Modeling the anatomical distribution of sunlight, Photochemistry and Photobiology, 79(1): 40 47, 2004

[13] Durnad F, A multidisciplinary survey of visibility, in ACM Siggraph course notes Visibility, Problems, Techniques, and Applications, July 2000.

[14] Cohen-Or D, Chrysanthou Y, Durand F, Greene N, Koltun V and Silva C, 2001. Visibility, problems, techniques and applications. SIGGRAPH Course Notes \# 30.

[15] Magnenat-Thalmann N and Thalmann D, Handbook of Virtual Humans, John Wiley and Sons Ltd (2004).

[16] MakeHuman, http://www.makehuman.org/.

[17] Meshlab, http://meshlab.sourceforge.net/.

[18] VCG, http://vcg.sourceforge.net.

[19] Poser, http:// www.e-frontier.com/.

[20] MakeHuman Project Abstract, http://www.dedalo3d.com/docs/articles/05_10_24_EN_MakeHuman_Proje ct_Abstract.pdf.

[21] Volz A, Blum R, Häberling S and Khakzar K, Automatic, body measurements based generation of individual avatars using highly adjustable linear transformation, HCI International 2007, Proceedings Volume 12, LNCS_4561, ISBN: 978-3-540-73318-8

[22] Di Giacomo T, Moccozet L, Magnenat-Thalmann N, Boulic R and Thalmann D, Automatic character skeletonization and deformation, State of the Art Report (STAR), Eurographics 2007, 2007.

[23] Magnenat-Thalmann N, Kalra P and Moccozet L, Virtual humans, in book HyperReality: Infra-structure for the Third Millennium, edited by N. Terashima and J. Tiffin, Routledge, ISBN:0415261031, 2001.

[24] Honglun H, Shouqian S and Yunhe P, Research on virtual human in ergonomic simulation, Computers \& Industrial Engineering, 53(2), 350-356, 2007.

[25] Sims EM, Reusable, lifelike virtual humans for mentoring and role-playing, Computers \& Education, 49(1):75-92, 2007

[26] Kiss B, Benedek B, Szijártó G, Csukly G, Simon L and Takács B, Virtual patient: a photo-real virtual human for VR-based therapy, Stud Health Technol Inform. 2004, 98:154-6.

[27] Seo $\mathrm{H}$ and Magnenat-Thalmann N, An example-based approach to human body manipulation, Graphical Models, 66 (1), 1-23, 2004

[28] Dirnberger J, Giretzlehner M, Ruhmer M, Haller H and Rodemund C., Modelling human burn injuries in a threedimensional virtual environment, Stud Health Technol Inform, 2003, 94:52-8.

[29] Xu XG, Taranenko V, Zhang J and Shi C, A boundaryrepresentation method for designing whole-body radiation dosimetry models: pregnant females at the ends of three gestational periods-RPI-P3, -P6 and -P9 2007 Phys. Med. Biol, 52, 7023-7044

[30] Abdel-Malek K, Human modeling and applications special issue, Computer-Aided Design Volume 39, Special Issue 7, Human Modeling and Applications, July 2007, Page 539.

[31] Dorward SE, A survey of object-space hidden surface removal, Internat J Comput Geom Appl 4 (1994), pp. 325-362.

[32] Bittner J and Wonka P, Visibility in Computer Graphics, Environment and Planning B: Planning and Design, 30(5):729-756, 2003.

[33] Downs NJ, Kimlin MG, Parisi AV and McGrath JJ, Modelling human facial UV exposure, 2000, Radiat. Prot. Aust. 17, 103-109.

[34] Holman CDJ, Gibson IM, Stephenson M and Armstrong ABK, 1983, Ultraviolet irradiation of human body sites in relation to occupation and outdoor activity: field studies using personal UVR, dosimeters. Clin. Exp. Dermatol. 8, 269-277. 